\title{
ASÍNTOTAS CURVAS EN FUNCIONES DEL PLANO CARTESIANO
}

\author{
Alberto Tirado Sanabria \\ aa.tirado@hotmail.com alberto.tirados@ug.edu.ec \\ Ciudad de Guayaquil, Ecuador. Código postal 090112
}

Recibido: 10 de octubre de 2020 Aceptado: 25 de mayo de 2021

\section{Resumen}

En la actualidad, los textos de matemáticas básica universitaria consideran la asíntota como una recta, en su deducción gráfica y analítica, como: vertical, horizontal y oblicua. Sólo en pocos ejemplos se menciona una asíntota curva cuadrática, como caso especial único sin deducción analítica, además poca mención se realiza en los casos cuando la asíntota es cortada por la función; es decir, con "toque o toques", pero con tendencia a la asíntota en valores extremos del dominio. Sin embargo, con el método: "Gráfica de Relaciones", o con un software para gráficas, se descubre que existen una infinidad de asíntotas curvas, algebraicas y transcendentes, donde su definición, deducción analítica y ejercitaciones tipo ejemplos, son los objetivos y resultados de este trabajo. Justificándose como novedad y ampliación al concepto vigente, entonces su relevancia está en el aporte teórico práctica a la primera matemática universitaria, en un rigor empírico para su demostración.

Palabras clave: Asíntotas curvas, tipologías, formulación y definiciones.

\section{ASYMPTOTES CURVES IN CARTESIAN PLANE FUNCTIONS}

\begin{abstract}
At present, basic university mathematics texts consider the asymptote as a line, in its graphical and analytical deduction, as: vertical, horizontal, and oblique. Only in a few examples is a quadratic curve asymptote mentioned, as a unique special case without analytic deduction, furthermore little mention is made in the cases when the asymptote is cut by the function; that is, with "touch or touches", but with a tendency to asymptote in extreme values of the domain. However, with the method: "Graph of Relationships", or with a software for graphs, it is discovered that there is an infinity of curved, algebraic, and transcendent asymptotes, where their definition, analytical deduction and exemplary exercises are the objectives and results. of this work. Justifying itself as a novelty and extension to the current concept, then its relevance is in the practical theoretical contribution to the first university mathematics, in an empirical rigor for its demonstration.
\end{abstract}

Keywords: Curved asymptotes, typologies, formulation, and definitions.

\section{ASÍNTOTAS CURVAS EM FUNÇÕES DO PLANO CARTESIANO}

\section{Resumo}

Atualmente, os textos de matemática universitária básica consideram a assíntota como uma linha, em sua dedução gráfica e analítica, como: vertical, horizontal e oblíqua. Apenas em alguns exemplos uma assíntota de curva quadrática é mencionada, como um único caso especial sem 
dedução analítica; além disso, pouca menção é feita nos casos em que a assíntota é cortada pela função; ou seja, com "toque ou toques", mas com tendência à assíntota em valores extremos do domínio. Porém, com o método: "Gráfico de Relações", ou com um software para gráficos, descobre-se que existe uma infinidade de assíntotas curvas, algébricas e transcendentes, onde sua definição, dedução analítica e exemplos de tipo de exercícios são os objetivos e resultados deste trabalho. Justificando-se como uma novidade e extensão do conceito atual, então sua relevância está na contribuição teórica e prática para a primeira matemática da universidade, em um rigor empírico para sua demonstração.

Palavras-chave: Assíntotas curvas, tipologias, formulação e definições.

\section{INTRODUCCIÓN}

Si dos funciones se operan por división, supongamos: $h(x)=f(x) / g(x)$, deben tomarse las siguientes consideraciones para $\mathrm{h}(\mathrm{x})$ como función cociente ${ }^{1}$

a) Si $\mathrm{f}(\mathrm{x})$ es diferente de cero, entonces la función denominador $\mathrm{g}(\mathrm{x})$ debe ser diferente de cero. De serlo el resultado de la función cociente $\mathrm{h}(\mathrm{x})$, es indefinido con tendencia al infinito en su imagen, el cual no es un número real; en estos casos se usa el decir: cuando $\mathrm{g}(\mathrm{x})$ tiende a cero, con $\mathrm{f}(\mathrm{x})$ diferente de cero, entonces $\mathrm{h}(\mathrm{x})$ tiende a infinito, en lo que se denomina asíntota vertical. b) $\operatorname{Si~} \mathrm{f}(\mathrm{x}) \mathrm{y} \mathrm{g}(\mathrm{x})$ ambas, son o tienden a cero para un mismo valor " $\mathrm{X}_{0}$ " dado, es una situación de indeterminación matemática, significando una tendencia desconocida, y se asume como el vacío de la función $\mathrm{h}(\mathrm{x})$ en dicho punto; es decir " $\mathrm{X}_{0}$ " no pertenece al dominio.

c) Si $\mathrm{f}(\mathrm{x}) \mathrm{y} g(\mathrm{x})$ toman valores al infinito y la función cociente $\mathrm{h}(\mathrm{x})$, tiende a un valor real, entonces puede resultar como el de una asíntota horizontal.

Hasta el momento y basado en los textos de matemáticas básicas, la deducción de las asíntotas se divide en solo dos: a) asíntota vertical, con el estudio de un límite lateral de la función cociente en un punto que anula el denominador; y b) asíntotas horizontal y oblicua, con el estudio de límites al infinito de la función cociente; esto debido a que es poco lo que se conoce sobre las asíntotas no rectas, por la ausencia de la gráfica de funciones en general en su enseñanza, (uso del método gráfico o de un paquete de computador con esta finalidad); hoy se desconoce la deducción analítica de esta tipología que denomino: "Asíntotas Curvas", (A.C)²; luego un concepto de asíntota de una función en el plano cartesiano actualmente es: el de una recta, a donde la función tiende a acercarse con ella, para valores específicos en el dominio de

\footnotetext{
${ }^{1}$ Con las funciones cocientes es que se inicia el llamado Cálculo I, pues a partir de aquí es que surgen las indefiniciones, las indeterminaciones y por supuesto las asíntotas.

${ }^{2}$ Primera definición, (el autor 2010).
} 
la función; del griego: "A - Sym - Totus", que significa: "sin coincidir", "que tiende a", "sin tocarlo". Entre otras definiciones de la actualidad, tenemos

1) "Es una recta a donde la función cociente se acerca". (Benítez, 2006), este autor en sus definiciones explica bajo la tutela de la aplicación del límite, las asíntotas horizontales, verticales u oblicuas.

2) "Definición analítica de una tendencia a una recta, en funciones cociente". (Ayres, 2003); este autor expone ejemplos gráficos sin expresión analítica.

3) "Es una recta que prolongada indefinidamente se acerca a una curva". (Santillana, 2010).

4) El autor (Purcell y otros 2001) define la asíntota como "Una recta a deducir con el estudio de límites infinitos y al infinito, para encontrar su aproximación a una función cociente”.

5) "Es una recta a donde la Gráfica de la función tiende", definición compartida en las obras de los autores: (Stewart 2008), (Edwars 1996) y (Swokwoski 1989). Estos autores de textos de Cálculo con geometría analítica aceptan la existencia de la asíntota curva $\mathrm{y}=\mathrm{x}^{2}$, como un caso especial y único para valores extremos en el dominio, de la función: $\left(x^{3}+1\right) / x$. Sin la deducción analítica de la misma.

Los objetivos de este trabajo son: mostrar la existencia de asíntotas curvas algebraicas en funciones del plano cartesiano, diferentes a la asíntota vertical que es una relación del plano definida como $\mathrm{X}=$ Número real. Su deducción empírica con el uso de ecuaciones propuestas del límite de una función, su confirmación por la noción tabular de una función y la ecuación definitiva posible para la deducción general de las asíntotas curvas; el concepto general de asíntota se predefine como: “Función algebraica a la cual, la función cociente tiende para valores extremos del dominio"3.

La idea es descubrir una serie de asíntotas curvas, que son funciones del plano, de funciones cocientes, por una estrategia inédita utilizando el límite al infinito, como una aplicación importante de este estudio matemático. Entendiendo que solo con la operación de división de funciones algebraicas y trascendentes trigonométricas, es que surgen las diferentes asíntotas, en un proceso empírico de descubrir posibles asíntotas curvas; en este desarrollo se emiten varios teoremas propios de esta investigación, como avance en el conocimiento sobre las asíntotas de funciones y como resultado propio de las ciencias y teorías de la educación sobre

${ }^{3}$ Considerada la primera ampliación lógica al concepto actual, al admitir la posibilidad superior de líneas en variadas formas como asíntotas, y no solo el de una recta, como en la actualidad, (el autor). 
este tema. En las funciones trascendentes logarítmicas y sus inversas exponenciales también pueden surgir asíntotas de cualquier índole por división, entre ellas o con otra función; sin embargo, en sus definiciones los Logaritmos: $\mathrm{y}=A \log _{B} X$ y las exponenciales: $\mathrm{y}=\mathrm{A} \cdot e^{F(x)}$, ya vienen con una asíntota recta característica: En la vertical negativa para las primeras y en la horizontal negativa para las segundas, para valores extremos.

Por supuesto el desarrollo ejemplar es y debe ser lo suficientemente amplio, como para no dejar dudas de su validez, en una finida de tipologías de funciones del plano y que le permiten al lector considerar este avance como una ampliación en la definición actual de la asíntota de una función, por un conjunto de definiciones descubiertas y que exigen de su conocimiento y posible ejercitación en toda matemática básica universitaria, llamada Matemáticas I o Cálculo diferencial. Luego por ser un trabajo de nuevas definiciones basado en resultados de ejercicios propuestos a continuación se hace un desarrollo empírico de 8 ejemplos escogidos en tipología, como básicos. El trabajo culmina con la nueva gama de definiciones que abarcan el concepto ampliado de la asíntota de una función.

\section{DESARROLLO EMPIRICO}

Para cumplir con el objetivo de descubrir las ecuaciones de las asíntotas curvas, inicia en la resolución de un ejemplo ilustrativo del concepto básico de asíntotas en la actualidad, luego se resuelven las situaciones que generan las nuevas concepciones y la evolución del concepto de asíntota: a) Con el cálculo del grado de la asíntota curva por una ecuación propia; b) Deducción que inicia por generalidad empírica, y concluye con las ecuaciones propuestas para la solución de las asíntotas curvas en funciones del plano cartesiano; y c) Realizar una propuesta de utilidad de estos conceptos en las matemáticas básicas universitarias ${ }^{4}$.

\footnotetext{
${ }^{4}$ Desde su desarrollo en el año 2010, he trabajado y calculado asíntotas curvas con los estudiantes que me asignan en secciones de Calculo I, así como en los talleres de formación docente, dictados.
} 
Ejercicio tipo ejemplo 1). Exprese las asíntotas de la función: $\mathrm{f}(\mathrm{x})=\left(\mathrm{x}^{2}+1\right) / \mathrm{x}$.

El dominio de la función $\mathrm{f}(\mathrm{x})$ es de todos los reales menos el valor en la horizontal de $\mathrm{x}=0$; esta recta es una asíntota vertical, el rango de la función es de: todos los reales menos el intervalo: $(-2,2)$, observación que se aprecia en la gráfica o con el cálculo analítico al despejar la "x" de la función que da este resultado. Figura 1. La tendencia de la función para valores muy grandes, positivos o

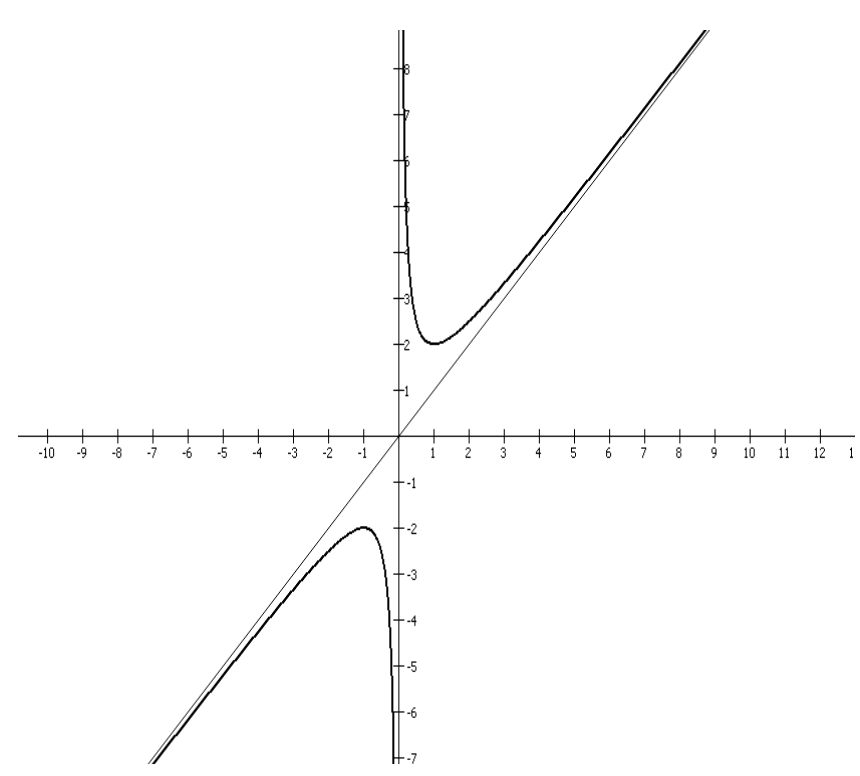
negativos es al mismo valor de la recta Figura 1: Función cociente $\mathbf{y}=\left(\mathbf{x}^{2}+1\right) / \mathbf{x}$. ver Tabla I, siguiente ${ }^{5}$ Expresada $y=x+1 / x$. Con su asíntota, $\mathbf{y}=\mathbf{x}$.

Tabla (I)

Valores de imágenes de: $y=\left(x^{2}+1\right) / x$; para valores dados del dominio.

\begin{tabular}{|c|c|c|c|c|c|}
\hline "x" & $f(x)$ & "x"x" & $f(x)$ & ${ }^{6} \mathrm{x} "$ & $\mathbf{f}(\mathbf{x})$ \\
\hline 5 & 5,2 & 100 & 100,01 & -10 & $-10,1$ \\
\hline 10 & 10,1 & 1.000 & $1.000,001$ & -20 & $-20,05$ \\
\hline 20 & 20,05 & -5 & -5.2 & -1.000 & $-1.000,001$ \\
\hline
\end{tabular}

La recta $\mathrm{y}=\mathrm{x}$, pareciera ser una asíntota recta inclinada llamada oblicua de la función del ejemplo; luego para el cálculo de los valores de la pendiente y del corte con la vertical de esta recta $y=a x+b$.

Se tiene que la pendiente se calcula como: $a=\lim _{x \rightarrow \pm \infty}(f(x) / x)$. Y con este valor se calcula el corte con la vertical como: $\mathrm{b}=\lim _{x \rightarrow \pm \infty}(\mathrm{f}(\mathrm{x})-\mathrm{a}(\mathrm{x}))$. (U.N.A, 2003) y (Benítez, 2006). Cuyos resultados son: $\mathrm{a}=1, \mathrm{y} \mathrm{b}=0$. Confirmando la tendencia en la noción tabular y lo que se observa a priori en la gráfica: la función tiende por encima $\mathrm{y}$ por debajo a la recta $\mathrm{y}=\mathrm{x}$, en valores extremos del dominio

\footnotetext{
${ }^{5}$ Noción tabular de una función, usada como estrategia lógica para descubrir tendencias o posibles imagenes por acercamiento infinitesimal en un punto o tendencias extremas en su dominio.
} 
Ejercicio tipo ejemplo 2). Exprese las asíntotas de la función: $f(x)=\left(x^{3}+1\right) / x$. como: $f(x)=$ $\left(x^{2}+1 / x\right)$.

Esta función tiene una asíntota vertical en la recta: $\mathrm{x}=0$, su dominio es: todos los números reales distintos de cero; el rango es todos los reales. Y de momento se observa cierta simetría, en referencia al eje vertical positivo en las dos curvas que "suben" a medida que los valores de " $x$ " se alejan del origen, cuando $x \rightarrow \pm \infty$. Esta observación sugiere que, sobre la función: $f(x)$ $=\left(x^{3}+1\right) / x$, hay la asíntota curva: $y=x^{2}$. Que se grafica en la figura 2, para demostrarlo; como también lo hacen (Stein, 1984) y (Thomas, 1998). A través de la noción tabular; ver tabla II.

Tabla (II)

Valores de imágenes de: $y=\left(x^{3}+1\right) / x$; para valores dados del dominio.

\begin{tabular}{|c|c|c|c|c|c|}
\hline & $f(x)$ & & $f(x)$ & "x" & $f(x)$ \\
\hline 5 & 25,2 & 50 & $2.500,02$ & -10 & 99,9 \\
\hline 10 & 100,1 & & 24,8 & -100 & $9.999,99$ \\
\hline
\end{tabular}

Se deduce una aproximación asintótica de la función dada, a la parábola: $\mathrm{y}=\mathrm{x}^{2}$, en valores altos; es decir que, para una tendencia a los infinitos, se aprecia visual y tabular que la función se acerca por encima a la parábola para valores positivos y por debajo, para valores negativos; en ambos casos con imágenes positivas $^{6}$. Estas situaciones abren un marco de posibilidades en el mundo de las asíntotas, en donde

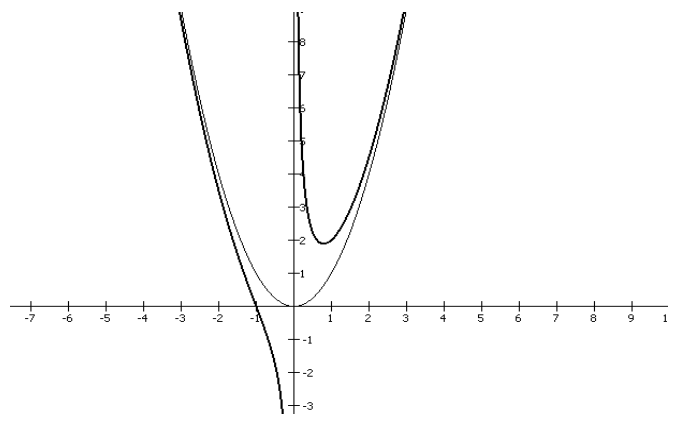

Figura 2: $f(x)=\left(x^{3}+1\right) / x$. Con $y=x^{2}$, como su asíntota curva. puede resultar que la línea en cuestión sea una función que haga de asíntota a una función cociente, y cuyo cálculo analítico de existencia queda por los momentos como un reto de índole matemático al nivel básico; es decir sin el uso de otras estrategias matemáticas superiores, como las conocidas series de convergencia, y transformadas.

\footnotetext{
${ }^{6}$ El lector debe saber que las graficas aqui realizadas se hicieron con un sotfware para este fin. Pero es virtud del método: "Grafica de relaciones", que la gráfica de toda función puede hacerse a mano por estudiantes y docentes, en el dominio de las operaciones con segmentos.
} 
Ejercicio tipo ejemplo 3). Exprese las asíntotas de la función: $f(x)=\left(x^{4}+1\right) / x$.

Esta función tiene una asíntota vertical en la recta: $\mathrm{x}=0$, su dominio es: todos los números reales menos el cero; el rango es todos los reales menos el intervalo abierto: $(-2,2)$. Y de momento se observa que, a medida que los valores de " $\mathrm{x}$ " se alejan del origen, la función tiende a la curva $x^{3}$. Esta observación sugiere que la función: $f(x)=\left(x^{4}+1\right) / x$, tiene por asíntota curva a la función $y=x^{3}$; que bien se puede asumir como solución visual en el marco de la novedad llamada: "Definición gráfica". Se puede además comparar esto, con la noción tabular de la función, donde adicionalmente se coloca una fila para la posible asíntota; ver Tabla III.

Tabla (III)

Valores de imágenes en: $y=\left(x^{4}+1\right)$ / $x$; para valores del dominio, con la posible asíntota ${ }^{7}$.

\begin{tabular}{|c|c|c|c|c|c|}
\hline${ }^{66} x "$ & $f(x)$ & $y=x^{3}$ & "x" & $f(x)$ & $y=x^{3}$ \\
\hline 4 & 64,25 & 64 & -5 & $-125,2$ & -125 \\
\hline 10 & $1.000,1$ & 1.000 & -20 & $8.000,05$ & 8.000 \\
\hline
\end{tabular}

Un avance ni siquiera al infinito, pero en otro valor superior como el número 100, nos ofrece el resultado de imagen en la función cociente de: 1.000.000,01 y en su asíntota de 1.000.000. Por lo que se deduce una aproximación asintótica para estos valores de la función a la curva cúbica en valores de tendencia extrema. Interesante es que en la gráfica se puede observar que la función cociente nunca "toca" a la función cúbica, posibilidad visual para valores extremos. Estos resultados (ejercicios 2 y 3), pueden apoyar la afirmación de que

Toda función que se sume a la función $\mathrm{y}=\mathrm{a} / \mathrm{x}^{\mathrm{n}}$, (Con " $\mathrm{n}$ " como número natural), resultará como una asíntota de la función suma: $\mathrm{f}(\mathrm{x})+\mathrm{a} / \mathrm{x}^{\mathrm{n}}$. Teorema $\mathrm{I}$, como primer aporte analítico de este trabajo.

Donde el grado de la asíntota curva, (A.C), resulta como

$\underline{\text { La operación inicial de restar el grado del numerador menos el grado del denominador, de una }}$ función algebraica cociente. Teorema II a manera de aporte analítico; Nota: Siempre y cuando

${ }^{7}$ En clases, y en los talleres realizados a docentes, esta tabla ampliada la llamo "Súper tabla", (el autor). 
el resultado de esta resta sea un número positivo y el numerador no sea una expresión algebraica del denominador ${ }^{8}$.

Deducción analítica en el ejemplo 2: La asíntota curva se sospecha como la función polinómica cuadrática: $y=x^{2}$. De grado dos: $y=a x^{2}+b x+c$.

Entonces por analogía del cálculo usado para una asíntota recta oblicua, se prueba lo siguiente

$$
\mathbf{a}=\lim _{x \rightarrow \pm \infty}\left(\mathbf{f}(\mathbf{x}) / \mathbf{x}^{2}\right) . \quad \mathbf{b}=\lim _{x \rightarrow \pm \infty}((\mathbf{f}(\mathbf{x}) / \mathbf{x})-\mathbf{a}(\mathbf{x})) . \quad \mathbf{c}=\lim _{x \rightarrow \pm \infty}\left(\mathbf{f}(\mathbf{x})-\mathbf{a}\left(\mathbf{x}^{2}\right)-\mathbf{b}(\mathbf{x})\right) .
$$

Estas ecuaciones arrojan como resultados: $\mathrm{a}=1, \mathrm{~b}=\mathrm{c}=0$. Es decir, la asíntota curva es: $\mathrm{y}=$ $x^{2}$.

Las ecuaciones resultan de dividir la función entre la variable " $\mathrm{x}$ " con el grado del polinomio de la asíntota para obtener el valor de "a, b y c", en una secuencia generalizada; veamos: Para "a" se divide en este caso entre $\mathrm{x}^{2}$, para el valor de " $\mathrm{b}$ " se divide la función entre el grado menor siguiente y se le resta el producto del valor de "a" por la variable "x", y para el valor de "c" se resta la función por el producto del valor obtenido de "a" por $\mathrm{x}^{2} \mathrm{y}$ del valor obtenido de "b" por $\mathrm{x}$.

El uso de estas ecuaciones y su generalización dependiente del problema, así como las aplicaciones en las matemáticas básicas universitarias son el aporte analítico de este artículo. Esta estrategia puede aplicarse para una asíntota de grado un número natural, iniciando en la utilidad del límite al : 1. Ver figura 3.

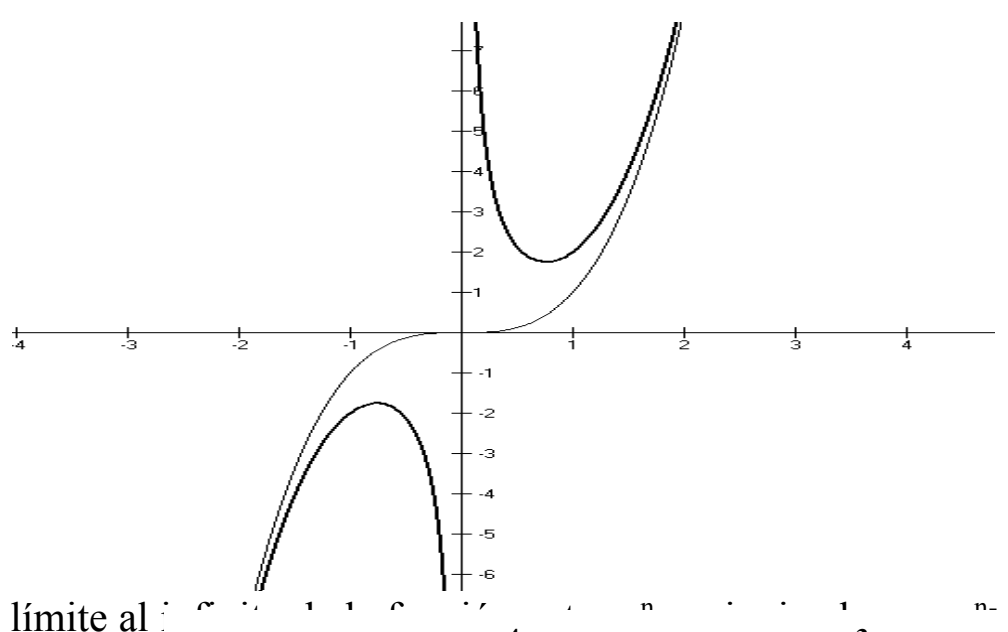
Figura 3: $f(x)=\left(x^{4}+1\right) / x$. Con $y=x^{3}$, como su asíntota.

Deducción analítica en el ejemplo 3: La asíntota es de grado tres, por: grados 4 - 1. Esto es del tipo cúbico, $\mathrm{y}=\mathrm{ax}^{3}+\mathrm{bx}^{2}+\mathrm{cx}+\mathrm{d}$. Entonces estos valores se obtienen por

\footnotetext{
${ }^{8} \mathrm{Si}$ la resta es igual a cero, la asintota es un recta horizontal, y de ser negativo; es decir el denominador es de grado mayor al numerador, entonces la asíntota es la recta horizonta: $\mathrm{y}=0$.
} 
$\mathbf{a}=\lim _{x \rightarrow \pm \infty}\left(\mathbf{f}(\mathbf{x}) / \mathbf{x}^{3}\right) ; \quad \mathbf{b}=\lim _{x \rightarrow \pm \infty}\left(\left(\mathbf{f}(\mathbf{x}) / \mathbf{x}^{2}\right)-\mathbf{a}(\mathbf{x})\right) ; \quad \mathbf{c}=\lim _{x \rightarrow \pm \infty}\left((\mathbf{f}(\mathbf{x}) / \mathbf{x})-\mathbf{a}\left(\mathbf{x}^{2}\right)-\mathbf{b}(\mathbf{x})\right)$ $\mathbf{d}=\lim _{x \rightarrow \pm \infty}\left(\mathbf{f}(\mathbf{x})-\mathbf{a}\left(\mathbf{x}^{3}\right)-\mathbf{b}\left(\mathbf{x}^{2}\right)-\mathbf{c}(\mathbf{x})\right)$. Respectivamente

De donde se obtiene los resultados: $\mathrm{a}=1, \mathrm{~b}=\mathrm{c}=\mathrm{d}=0$; es decir la asíntota es la función: $\mathrm{y}=$ $\mathrm{x}^{3}$.

Véase los siguientes 5 ejercicios a manera de ejemplos, en deducción empírica.

Ejercicio tipo ejemplo 4). Exprese las asíntotas de: $f(x)=x^{3}+\left(1 / x^{2}\right)=\left(x^{5}+1\right) / x^{2}$. En este ejemplo la asíntota resultaría como: $\mathrm{y}=\mathrm{ax}^{3}+\mathrm{bx}^{2}+\mathrm{cx}+\mathrm{d}$. de grado tres por $5-2$. Y al aplicarle el cálculo de estos valores se tiene que
$\mathbf{a}=\lim _{x \rightarrow \pm \infty}\left(\left(x^{5}+1\right) / \mathbf{x}^{5}\right) .=1$
$\mathbf{b}=\lim _{x \rightarrow \pm \infty}\left(\left(\mathbf{x}^{5}+1-\mathbf{x}^{5}\right) / \mathbf{x}^{4}\right)=\mathbf{0}$.
$\mathbf{c}=\lim _{x \rightarrow \pm \infty}\left(\left(\mathbf{x}^{5}+1-\mathbf{x}^{5}\right) / \mathbf{x}^{3}\right)=\mathbf{0}$.
$\mathbf{d}=\lim _{x \rightarrow \pm \infty}\left(\left(\mathbf{x}^{5}+1-\mathbf{x}^{5}\right) / \mathbf{x}^{2}\right)=\mathbf{0}$.

Es decir, la asíntota resulta como la curva: y $=x^{3}$, que es el resultado que se observa en la gráfica. Y se puede confirmar con la noción tabular de la función. Digamos que $\mathrm{x}=-3$ entonces la imagen es $\mathrm{f}(-3)=-26,8889 \mathrm{y}$ en la cúbica en $\mathrm{x}=-3$, el resultado es -27 ; luego si $x=-20$ entonces en la función cociente, $\mathrm{f}(-$ 20) $=-7.999,9975$ y en la cúbica en $x=-20$ el resultado es 8.000, por lo que se comprueba una aproximación asintótica de la

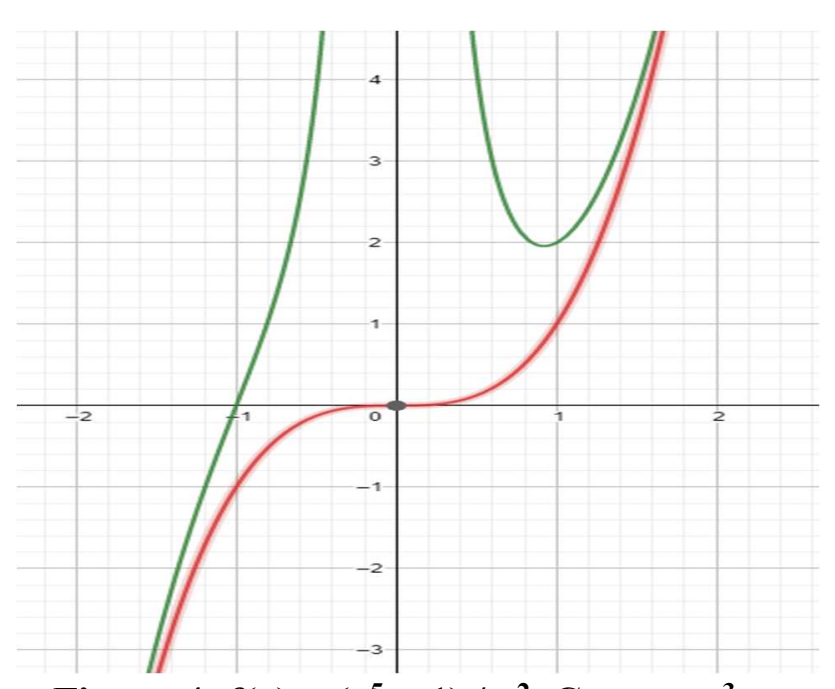

Figura 4: $f(x)=\left(x^{5}+1\right) / x^{2}$. Con $y=x^{3} \operatorname{comosu}($ A.C).

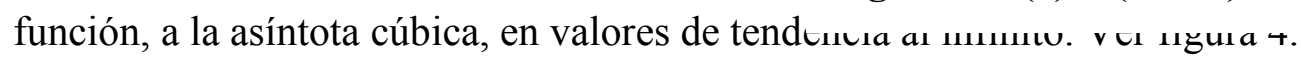

Ejercicio tipo ejemplo 5). Exprese las asíntotas de la función: $f(x)=\left(2 x^{4}+x^{3}-2 x+1\right) / x$. Esta función tiene una asíntota vertical en la recta: $\mathrm{x}=0$, su dominio es: todos los números reales sin el cero; el rango como la unión de los intervalos, aproximado: $(-\infty ;-3,5) \cup(0,4 ;+\infty)$; que se observa, pues su confirmación analítica no es sencilla. Para confirmar, se aplica las ecuaciones deducidas para una asíntota: $y=a x^{3}+b x^{2}+c x+d$. por grado de $4-1$. Que ofrece el siguiente resultado 
$\mathbf{a}=\lim _{x \rightarrow \pm \infty}\left(\left(2 x^{4}+x^{3}-2 x+1\right) / x^{4}\right) .=2 . \quad b=\lim _{x \rightarrow \pm \infty}\left(\left(\left(2 x^{4}+x^{3}-2 x+1\right) / x^{3}\right)-2 x\right)=1$.

$\mathbf{c}=\lim _{x \rightarrow \pm \infty}\left(\left(\left(2 x^{4}+\mathbf{x}^{3}-2 x+1\right) / \mathbf{x}^{2}\right)-2 x^{2}-\mathbf{x}\right)=0 . \quad \mathbf{d}=\lim _{x \rightarrow \pm \infty}\left(\left(\left(2 x^{4}+\mathbf{x}^{3}-2 x+1\right) / \mathbf{x}\right)-2 x^{3}-\right.$ $\left.\mathbf{x}^{2}\right)=-2$.

La función $y=2 x^{3}+x^{2}-2$, que también se gráfica, es la asíntota curva de $\mathrm{f}(\mathrm{x})$, a medida que los valores de " $x$ " se alejan del origen. Ver figura 5.

El lector puede si quiere realizar la confirmación por la noción tabular de una función para valores dados. Una "Súper tabla", de donde aparezca la tendencia, la función y la asíntota calculada.

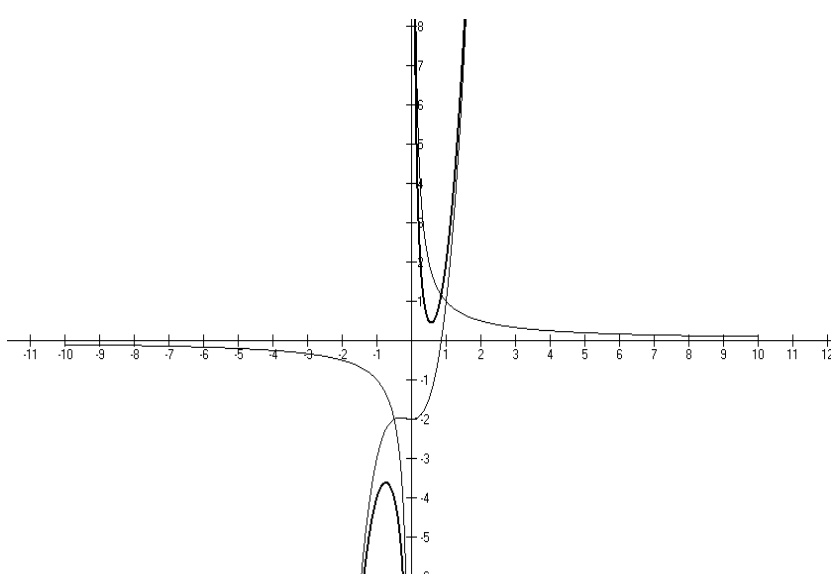

Figura 5: $f(x)=2 x^{3}+x^{2}-2+1 / x$. Con su A.C: $y=2 x^{3}+x^{2}-2$.

Ejercicio tipo ejemplo 6). Exprese las asíntotas de la función cociente: $f(x)=|x|+1 / x$. En este ejemplo la función posee una asíntota vertical en el origen y una asíntota recta oblicua o inclinada: $\mathrm{y}=\mathrm{ax}+\mathrm{b}$. Donde al aplicarle el cálculo de estos valores por la ecuación de una asíntota oblicua, para valores positivos y negativos, tienen los siguientes resultados

$$
\mathrm{a}=\lim _{x \rightarrow \pm \infty}\left((\mathrm{x} .|\mathrm{x}|+1) / \mathrm{x}^{2}\right)= \pm 1 . \quad \mathrm{b}=\lim _{x \rightarrow \pm \infty}\left(\left(\mathrm{x} .|\mathrm{x}|+1 \pm \mathrm{x}^{2}\right) / \mathrm{x}\right)=0 .
$$

Luego, la asíntota resulta como: $y=|x|$, que es el resultado que se observa en la gráfica. Ver figura 6, donde se aprecia los sumandos y la función suma: $\mathrm{f}(\mathrm{x})=|\mathrm{x}|+1 / \mathrm{x}$. El lector, puede si quiere, realizar la confirmación por la noción tabular de una función para valores extremos dados en el dominio.

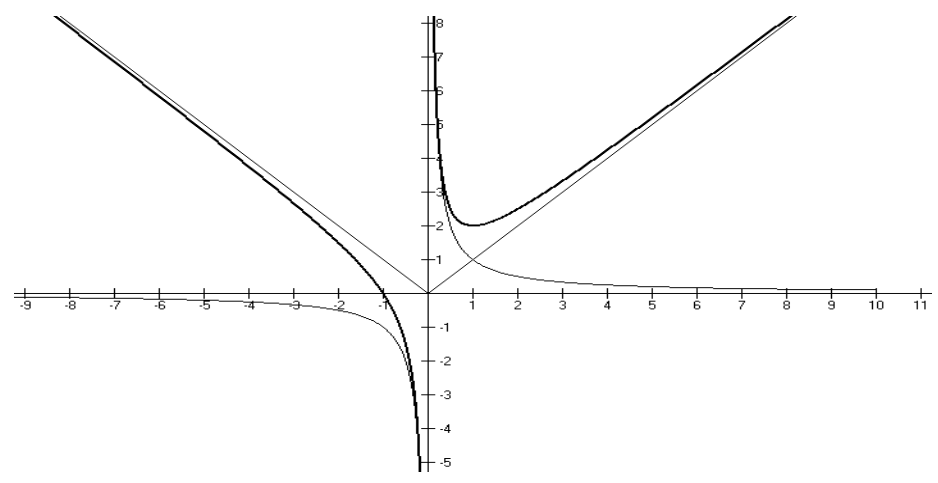

Figura 6: $f(x)=|x|+1 / x$. Donde $y=|x|$ es su asíntota oblicua. 
Ejercicio tipo ejemplo 7). Exprese las asíntotas de la función: $\mathrm{f}(\mathrm{x})=\sqrt{x}+1 / \mathrm{x}=(\mathrm{x} \cdot \sqrt{x}+1) / \mathrm{x}$. La función posee una asíntota vertical en el origen y una asíntota curva como: $\mathrm{y}=\mathrm{a} \sqrt{x}+\mathrm{b}$. Esto por el grado de $1,5-1=1 / 2$. Y al aplicarle el cálculo de estos valores por la ecuación deducida de una asíntota curva, tienen el siguiente resultado

$$
\mathrm{a}=\lim _{x \rightarrow \pm \infty}((\mathrm{x} \cdot \sqrt{x}+1) / \mathrm{x} \cdot \sqrt{x})=1 . \quad \mathrm{b}=\lim _{x \rightarrow \pm \infty}((\mathrm{x} \cdot \sqrt{x}+1-\mathrm{x} \cdot \sqrt{x}) / \mathrm{x})=0 .
$$

La asíntota curva es: $\mathrm{y}=\sqrt{x}$; para valores extremos positivos en el dominio de $\mathrm{f}(\mathrm{x})=(\mathrm{x} \sqrt{x}+1) / \mathrm{x}$. Que es el resultado observado en la gráfica de esta función cociente. Ver figura 7.

Nuevamente el lector puede si quiere, realizar la confirmación por la noción tabular de una función para valores extremos dados.

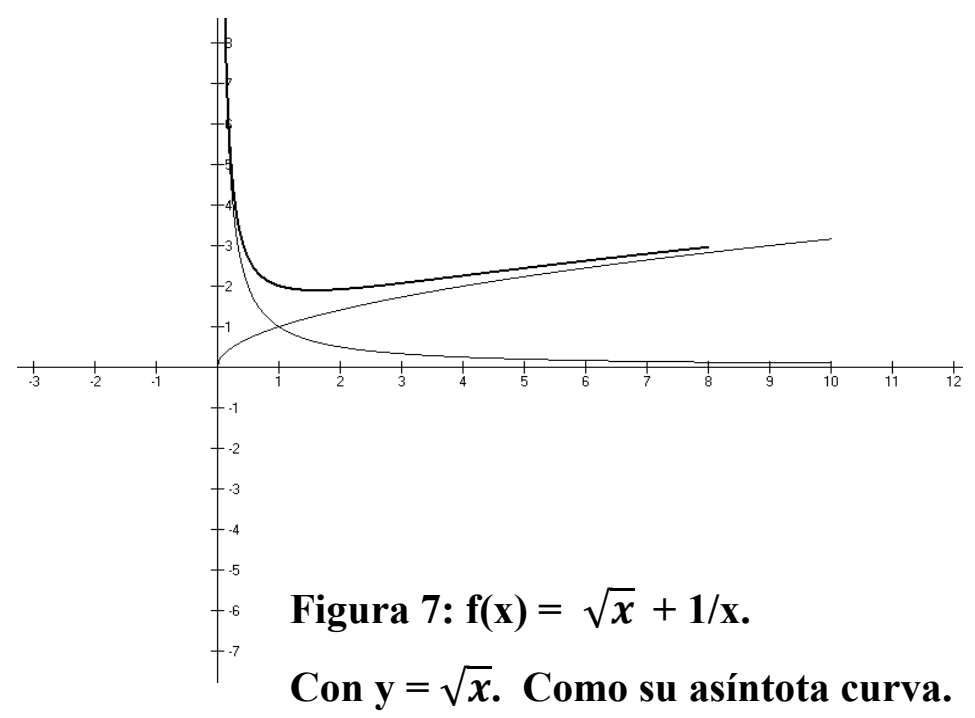

Ejercicio tipo ejemplo 8). Exprese las asíntotas de la función cociente: $\mathrm{f}(\mathrm{x})=\left(\mathrm{x}^{3}+\operatorname{Cos}(\mathrm{x})\right) / \mathrm{x}$. En este ejemplo la función posee una asíntota vertical en el origen y una asíntota curva como la cuadrática: $\mathrm{y}=\mathrm{ax}^{2}+\mathrm{bx}+\mathrm{c}$. Y al aplicarle el cálculo de estos valores por la ecuación deducida de una asíntota curva, se obtiene el resultado

$$
\begin{aligned}
& \left.\mathbf{a}=\lim _{x \rightarrow \pm \infty}\left(\mathbf{x}^{3}+\operatorname{Cos}(\mathbf{x})\right) / \mathbf{x}^{3}\right)=1 . \quad \mathbf{b}=\lim _{x \rightarrow \pm \infty}\left(\left(\mathbf{x}^{3}+\operatorname{Cos}(\mathbf{x})-\mathbf{x}^{3}\right) / \mathbf{x}^{2}\right)=\mathbf{0} . \\
& \mathbf{c}=\lim _{x \rightarrow \pm \infty}\left(\left(\mathbf{x}^{3}+\operatorname{Cos}(\mathbf{x})-\mathbf{x}^{3}\right) / \mathbf{x}\right)=\mathbf{0} .
\end{aligned}
$$


Entonces, la asíntota curva resulta como: $y=x^{2}$; para valores extremos en el dominio de esta función trigonométrica; que es el resultado que se observa en la gráfica. Con el adicional que la asíntota es cortada por la función en periódicos puntos específicos, antes de "confundirse" con ella para valores extremos en el dominio. El hecho del corte con la asíntota en algunos puntos determinados en el dominio abre una serie de posibilidades tipológicas en el sentido del concepto original de las asíntotas, (Sin toque); es por ello por lo que aquí se define estas tipologías particulares de funciones con el nombre de "Síntotas" es decir, en lenguaje original griego: "Con Toque", significa que es una asíntota en valores extremos, pero la función cociente la toca

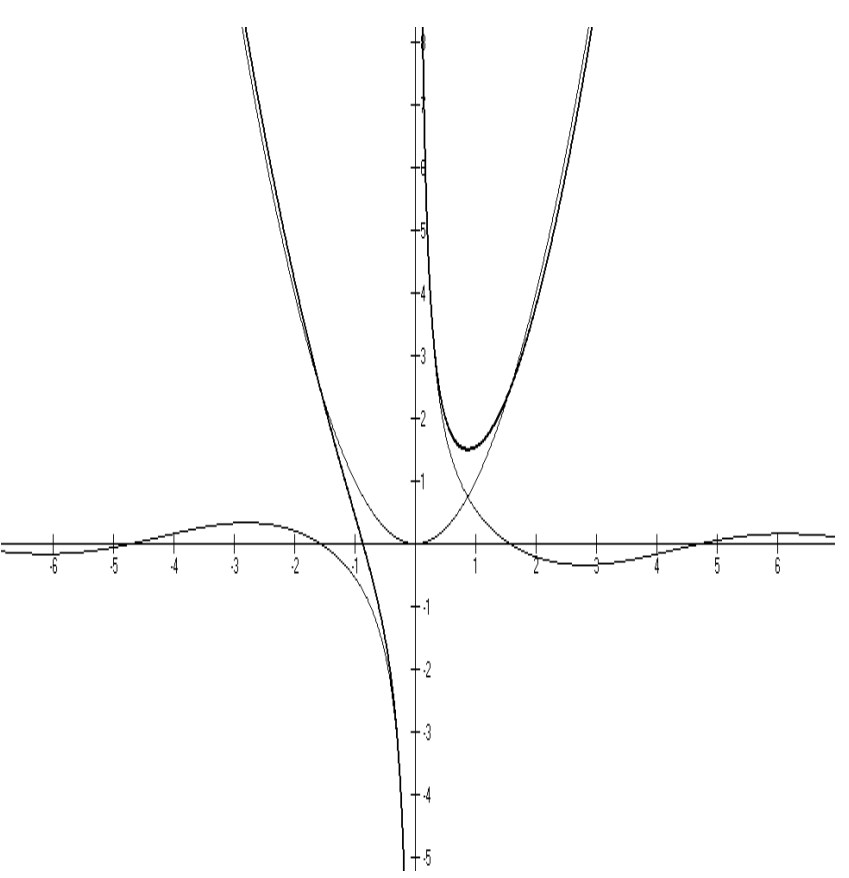

Figura 8: $f(x)=x^{2}+\operatorname{Cos}(x) / x$.

Donde $y=x^{2}$. Es su "Síntota" curva o intercepta en puntos específicos dados ${ }^{9}$. Figura 8 .

El resultado en este ejemplo conduce a la afirmación de lo que aquí se denomina el teorema III Toda función algebraica que se sume a la función $\mathrm{y}=\operatorname{Cos}(\mathrm{x}) / \mathrm{x} \quad \mathrm{o} \quad \mathrm{y}=\operatorname{Sen}(\mathrm{x}) / \mathrm{x}$, resultara en $\underline{\text { sí misma, como una asíntota de esa, función suma }}{ }^{10}$.

En variedades bibliográficas matemáticas, existen graficas referenciales de funciones algebraicas cocientes donde se aprecian los cortes de la función con su asíntota, (sin un estudio respectivo sobre este o estos puntos), y solo como en casos de asíntotas horizontales o inclinadas, como en problemas del tema de funciones en los textos de: (Giudici, 2008); (Purcell y otros 2001) y (Benítez 2006). Todos sin ejemplos de asíntotas en funciones transcendentes. A continuación, algunos ejercicios propuestos sobre lo visto, y sobre el tema innovador de las posibles operaciones algebraicas entre asíntotas.

\footnotetext{
${ }^{9}$ Esta palabra no existe en la actualidad en la lengua castellana.

${ }^{10}$ Aceptando que en la primera opción hay una asíntota vertical en el origen, y en la segunda función, la del seno, en el origen, lo que hay es un vacio o punto indeterminado, con tendencia a la unidad.
} 
Ejercicios propuestos, con su respuesta a verificar, analítica o por noción tabular

1) $f(x)=\frac{(x+1)^{2}}{(x-1)^{2}} \quad \mathbf{R}:$ Asíntota vertical en $x=1$. Sintota horizontal: $y=1$, con corte en $\mathrm{x}=0$.

2) $f(x)=\frac{x^{4}}{(x+1)^{2}} \quad$ R: A.V en $x=-1$. Sintota curva: $y=(x-1)^{2}$, con cortes en $x= \pm \sqrt{0,5}$.

3) $\mathrm{f}(\mathrm{x})=\frac{x^{2}}{4}+\frac{\operatorname{Sen}(x)}{x}$. R: Sintota curva: $\mathrm{y}=\left(\frac{x}{2}\right)^{2}$, con cortes en los múltiplos enteros de $\pi$.

4) $\mathrm{f}(\mathrm{x})=\frac{(x-1)^{3}}{x}$. R: Asíntota vertical en $\mathrm{x}=0$. Asíntota curva: $\mathrm{y}=\mathrm{x}^{2}-3 \mathrm{x}+3$.

5) $\mathrm{f}(\mathrm{x})=\frac{(x-1)^{6}}{x^{2}}$. Función que resulta de elevar la anterior al cuadrado, es decir, en producto por sí misma. R: Asíntota vertical en $x=0$. Síntota curva: $y=x^{4}-6 x^{3}+15 x^{2}$ $-18 x+9$, para valores extremos en ambos lados del dominio y con corte único en un punto cercano al valor $\mathrm{x}=0,6$.

Esta sintota curva, es una función que se obtiene de elevar al cuadrado la asíntota del problema 4 anterior ${ }^{11}$.

\section{RESULTADOS Y SUS ANALÍSIS}

El resultado del trabajo es el hecho de la existencia de las asíntotas curvas en funciones cocientes del plano cartesiano, asíntotas que son representadas por otras funciones; estas se pueden obtener por la aplicación del límite al infinito, en una ampliación del uso actual, deducido como avance del conocimiento en el ejemplo 2. Además de los teoremas I y II, como estrategias agregadas para el grado de la asíntota algebraica, como el hecho notable de: la suma entre una función cociente cualquiera con una función del tipo $\mathrm{f}(\mathrm{x})=1 / \mathrm{x}^{\mathrm{n}}$, con $(\mathrm{n})$, como número natural como se dijo.

Otro resultado de interés matemático está, en el hecho de establecer si es con "Toque" o "Toques", para puntos definidos del dominio y siempre asintótica para valores extremos; el cálculo de estos puntos específicos se obtiene con el despeje de la igualación de la asíntota con la función cociente; donde incluso de no resultar una ecuación algebraica sencilla, puede

\footnotetext{
${ }^{11}$ Interesante este hecho donde la sintota obtenida resulta en formula como el producto por si misma de la asíntota presedente, por el hecho de que la función cociente se elevo al cuadrado. Entonces ¿Se pueden hacer teorizaciones al respecto, sobre el movivmiento de las asintotas, por operaciones algebraicas?
} 
estudiarse con límites indeterminados o con aproximación infinitesimal tabular, para obtener este interesante punto del dominio. Por último, el solo hecho de realizar operaciones algebraicas con funciones cocientes, como producto o diferentes potencias, ocasiona que existe un "movimiento" en la asíntota original, (diferente a la asíntota vertical que es incólume), ocasionando según la operación en otra asíntota recta o en una asíntota curva; todas ellas sin contar con teoremas de resultados, en la actualidad ${ }^{12}$.

Todo esto ofrece el siguiente avance conceptual en las asíntotas en general, que se puede definir como: "Una línea en donde la función cociente tiende a ella, para valores puntuales y extremos en el dominio", en las siguientes tipologías: 1) Asíntota vertical: como una recta vertical a donde la función cociente tiende para valores puntuales en el dominio; donde el límite de la función es de resultado infinito denominado indefinido. 2) Asíntota horizontal: como una o dos rectas horizontales hacia los infinitos, a donde la función cociente tiende para valores extremos del dominio; donde el límite al infinito de la función tiene por resultado un número real. 3) Asíntota oblicua: como una o dos rectas inclinadas en sentido de los infinitos, a donde la función cociente tiende para valores extremos del dominio; cuya deducción requiere del límite al infinito, en la estrategia actual conocida de descubrir su pendiente con $f(x) / x, y$ de existir se calcula su corte en $f(x)-a(x)$. 4) Asíntota curva: como una función algebraica o trascendente, donde la función cociente tiende para valores extremos del dominio; cuya deducción y grado provienen de los teoremas y deducciones expuestos en este trabajo en la generalización del estudio del límite al infinito de la función. 5) Sintota: es toda asíntota diferente a la vertical, donde la función cociente tiende para valores extremos, donde pueden ocurrir intercepciones en uno, varios o infinitos puntos conocidos del dominio.

\section{DISCUSIÓN E IMPLICACIONES PEDAGÓGICAS}

El aporte en el avance del conocimiento principal de esta investigación está, en que demuestra la existencia y deducción de asíntotas curvas o representadas por otras funciones del plano, diferente a la función cociente. Existencia que puede y debe ser estudiada en la matemática básica universitaria, en al menos sus existencias, o como avance en análisis matemático en sus deducciones y teoremas donde se exige una importante ampliación del concepto actual; en la nueva definición general de asíntotas en las tipologías expuestas.

\footnotetext{
${ }^{12}$ En innumerables ejercicios realizados, he llegado a la conclusión de que se pueden generar teoremas al respecto, sobre el movimiento de las asíntotas no verticales. (El autor).
} 
De hecho, el planteamiento como la posibilidad lógica en la existencia de las asíntotas representadas por funciones transcendentes, de otras funciones cociente demuestra que la propuesta tiene valor y consecuencias dignas de investigar, en el mundo de la matemática inicial universitaria; donde este trabajo genera el debate y posibilidad de realizar el estudio para normalizar las ecuaciones propuestas en adelante con el uso de la simbología de la sumatoria o con alguna otra aplicación algebraica, para expresarlas analíticamente, con la formalidad matemática requerida.

En definitiva, el uso del método "Grafica de Relaciones" es un elemento didáctico importante en la enseñanza de la matemática básica, que resulta en una retroalimentación del concepto; la gráfica de las funciones, manual o asistido con un software para este fin permite "Ver" sus características y propiedades, a la vez que ofrece el aporte de la comparación y confirmación de resultados analíticos y tabulares.

A nivel de la primera o segunda matemática universitaria, el descubrir las llamadas ahora "Asíntotas curvas" es una posibilidad ejemplar en las aplicaciones del límite de una función, e incluso como novedad en el mundo de la matemática numérica por aproximaciones, entre estas posibilidades tenemos: 1) Investigar sobre el uso y deducción de estos conceptos en la enseñanza de la matemática básica universitaria, que contengan el tema de la aplicación del límite de una función, como didáctica aplicada en la pedagogía matemática. 2) Con los resultados que se señalan queda como reto al investigador la demostración de la deducción propuesta y el descubrir la deducción analítica de las asíntotas curvas trascendentes, en la formalidad matemática, 3) Estudiar la existencia general de "Síntotas" rectas o curvas en funciones algebraicas o trascendentes del plano cartesiano, es de interés y un estudio particular del límite de una función, pueden entonces descubrirse teoremas que permitan a priori, afirmar la existencia de estas "Con toque", por el grado y los coeficientes de las funciones a dividir.

\section{REFERENCIAS}

AYRES, F. \& MENDELSON, E. (2003). Cálculo. Cuarta edición. Editorial, Mc Graw Hill. Colombia.

BENÍTEZ, R. (2006). Cálculo diferencial. Primera edición. Editorial Trillas. Ciudad de México (pp. 169-181).

EDWARS, C. \& PENNEY, D. (1996). Cálculo con geometría analítica. Cuarta edición. Editorial, Prentice Hall. México. 
GIUDICI, R. / GIUDICI R. (2008). Guía de problemas matemáticas 1. Editorial Equinoccio. Dirección de cultura de la Universidad Simón Bolívar. Venezuela (pp. 234-244).

PURCELL E. / VARBERG D. / RIGDON S. (2001). Cálculo $8^{\mathrm{va}}$ Edición. Prentice Hall. México.

SANTILLANA. (2010). Matemática 2, edición del docente. Segunda edición. Editorial McGraw-Hill. Ciudad de México (pp. 152 Glosario).

STEIN, S. (1984). Cálculo y geometría analítica. Tercera edición. Editorial McGraw-Hill. México (pp. 71-76)

STEWART, J. (2008). Cálculo de una variable. Sexta edición. Editorial Cengage Learning. México.

SWOKWOSKI, E. (1989). Cálculo con geometría analítica. Segunda edición. Editorial Iberoamericana. Colombia.

THOMAS G. \& FINNEY R. (1998). Cálculo en una variable. Novena edición. Editorial, Addison Wesley. México.

UNIVERSIDAD NACIONAL ABIERTA (2003). Matemática II, modulo I. Código 178-179. Vicerrectorado académico, matemáticas, ISBN 980-236-588-9. Caracas (pp. 86-95). Venezuela.

Autor:

Alberto Antonio Tirado Sanabria

(UG-Ecuador), Universidad de Guayaquil - Ecuador

Ingeniero Industrial, Universidad de Oriente, Núcleo de Anzoátegui, en 1997. Máster en educación, mención enseñanza de la Matemática Básica Universidad de Oriente. 2010. Autor de los textos: Mecánica Clásica para estudiantes de Ingeniería, Física I" 2 da edición del 2015 "Calculo Diferencial" en término del borrador final "Manual para la prueba Ser Bachiller" en arbitraje para su publicación, por la U.G. Profesor Contratado, en la Universidad de Guayaquil. Ecuador. Facultad de Ciencias Matemáticas y Físicas, 2018. Alberto.tirados@ug.edu.ec; aa.tirado@,hotmail.com 